\title{
High Dynamic Range Imaging of Non-Static Scenes
}

\author{
Imtiaz Hossain and Bahadir K. Gunturk \\ Dept. of Electrical and Computer Engineering \\ Louisiana State University, Baton Rouge, LA 70803
}

\begin{abstract}
A well-known technique in high dynamic range (HDR) imaging is to take multiple photographs, each one with a different exposure time, and then combine them to produce an HDR image. Unless the scene is static and the camera position is fixed, this process creates the so-called "ghosting" artifacts. In order to handle non-static scenes or moving camera, images have to be spatially registered. This is a challenging problem because most optical flow estimation algorithm depends on the constant brightness assumption, which is obviously not the case in HDR imaging. In this paper, we present an algorithm to estimate the dense motion field in image sequences with photometric variations. In an alternating optimization scheme, the algorithm estimates both the dense motion field and the photometric mapping. As a latent information, the occluded regions are extracted and excluded from the photometric mapping estimation. We include experiments with both synthetic and real imagery to demonstrate the efficacy of the proposed algorithm. We show that the ghosting artifacts are reduced significantly in HDR imaging of non-static scenes.
\end{abstract}

Keywords: High dynamic range imaging, photometric registration, optical flow estimation

\section{INTRODUCTION}

The dynamic range of a real world scene can be much higher than a camera can capture. A well-known technique to create a high dynamic range (HDR) image is to take multiple photographs with different exposure times (or ISO speeds) and then combine them to estimate the irradiance of the scene. ${ }^{1}$ This requires a static scene and a fixed camera position; otherwise, misalignments of images or occlusions may create objectionable artifacts, which typically appear as faint regions and therefore are called "ghosting" artifacts. There are three main approaches to produce an artifact-free HDR image:

- The first approach is to take the best exposed region from the stack of images available and in a sense form a collage of best exposed regions. ${ }^{2,3}$ There are some major issues associated with this approach. Objects that are moving in the scene could appear in multiple locations or deformed; also, there could be discontinuities or artifacts along the region boundaries.

- The second approach is to take a weighted sum of images, where the weights are determined such that pixels likely to be belonging to a moving object are given less weight. ${ }^{1,4,5}$ This reduces ghosting artifacts; on the other hand, since moving objects are not incorporated into the HDR creation, not all available information is used in the process and therefore the best possible dynamic range enhancement is not achieved.

- The third approach is to estimate the motion field and create the HDR image after compensating for the motion. Although this seems to be the best approach, the major challenge is the estimation of the motion field. Standard optical flow algorithms would not work properly because the constant brightness assumption is not valid anymore as images are captured with different exposure times; images have to be photometrically registered first. On the other hand, most photometric registration algorithms require that images are geometrically registered (motion compensated). We can group the solutions to this problem in three categories: (i) Photometrically register images using an algorithm that can handle misalignments of images, and then apply an optical flow estimation algorithm. (ii) Geometrically register images using an algorithm that is insensitive to photometric differences, and then apply a photometric registration algorithm. (iii) Apply a joint photometric and geometric registration algorithm.

\footnotetext{
Contact information: imtiaz@lsu.edu and bahadir@ece.lsu.edu.
} 
In the first category, one can utilize the histogram based photometric registration proposed by Grossberg and Nayar. ${ }^{6}$ Although this algorithm can handle cases where the images overlap largely and exposure times are close, occlusion and saturation may degrade the accuracy of photometric mapping estimation. In the second category, the illumination invariant feature extraction method of Gevrekci and Gunturk ${ }^{7}$ is a possible algorithm to extract the interest points in the images and finally produce a parametric motion field. Another possible algorithm is proposed by Kharbat et al. ${ }^{8}$ where, instead of brightness constancy, normalized geometric moment constancy is used to estimate the motion field. The idea is based on the fact that the normalized geometric moment is insensitive to brightness changes. In the third category, there are a number of algorithms in the literature. For example, Baker and Matthews ${ }^{9}$ and Bartoli ${ }^{10}$ model photometric mapping as gain and offset terms and estimate the motion field as well the photometric mapping parameters in a way inspired by the Lucas-Kanade approach. ${ }^{11}$ Similarly, Kim et al. ${ }^{12-14}$ models photometric mapping as space-varying gain and offset mappings ${ }^{15,16}$ and estimates the dense motion field and photometric transformation in an iterative scheme similar to the Horn-Schunck approach. ${ }^{17}$ Instead of estimating the dense motion field, parametric modeling of the motion field is also possible. ${ }^{18-20}$ A recent approach by Fouad et al. ${ }^{21}$ proposes an affine sub-pixel registration method combined with a segmentation based local illumination model that considers a number of arbitrary linear functions as candidate illumination functions at each pixel. The number of such functions corresponds to the number of possible different illumination levels in two images. For real world images where the illumination levels in the input images are not known a priori, an iterative k-means based segmentation is suggested.

In this paper, we present a method that also falls in the third category. The photometric mapping is modeled as a global non-parametric transformation, which accurately models photometric variations due to camera exposure changes. The proposed method estimates the dense motion field and the photometric mapping in an alternating optimization framework. During the iterations, unreliable (e.g., occluded) pixels are also estimated and excluded from the photometric estimation.

\section{DENSE MOTION FIELD AND PHOTOMETRIC MAPPING ESTIMATION}

According to the constant brightness assumption, two images $I_{1}$ and $I_{2}$ are related as $I_{1}(\mathbf{x}+\mathbf{u}(\mathbf{x}))=I_{2}(\mathbf{x})$, where $\mathbf{x}=(x, y)$ is a pixel position and $\mathbf{u}(\mathbf{x})=(u(\mathbf{x}), v(\mathbf{x}))$ is the motion vector at that pixel. If there is also a photometric change between the images, then the constant brightness optical flow equation should be modified to reflect that. The photometric mapping can be incorporated in two ways:

$$
g_{21}\left(I_{1}(\mathbf{x}+\mathbf{u}(\mathbf{x}))\right)=I_{2}(\mathbf{x})
$$

and

$$
I_{1}(\mathbf{x}+\mathbf{u}(\mathbf{x}))=g_{12}\left(I_{2}(\mathbf{x})\right),
$$

where $g_{21}$ is the intensity mapping function (IMF) from $I_{1}$ to $I_{2}$, and $g_{12}$ is the IMF from $I_{2}$ to $I_{1}$. It is important to note that these equations may lose their validity for some pixels: Apart from the relatively small quantization error and noise, saturation and occlusion lead to pixels that deviate significantly from (1) and (2). The visibility of pixels is directional, that is, it depends on the direction of the photometric mapping. While it is possible to obtain a pixel value in one image from the other image through photometric mapping, the other way around may not be possible. Therefore, we should be explicit about the direction of the photometric mapping and the error as a result of the mapping. Here, we define $r_{1}$ and $r_{2}$ as the residuals from photometric mappings of $I_{1}$ and $I_{2}$ :

$$
r_{1}(\mathbf{x})=I_{2}(\mathbf{x}-\mathbf{u}(\mathbf{x}))-g_{21}\left(I_{1}(\mathbf{x})\right)
$$

and

$$
r_{2}(\mathbf{x})=I_{1}(\mathbf{x}+\mathbf{u}(\mathbf{x}))-g_{12}\left(I_{2}(\mathbf{x})\right),
$$

where, in (3), $I_{1}$ is photometrically mapped to match $I_{2}$, and $I_{2}$ is geometrically registered to $I_{1}$. In (4), the mappings are the other way around.

We would like to find the motion field and the photometric mappings that minimize the residuals. We adopt an iterative scheme to achieve that. Starting with an initial estimate for the motion field (specifically $\mathbf{u}(\mathbf{x})=\mathbf{0}$ ), we first calculate the photometric mappings; and then, with the new photometric mappings, we update the 
motion field. The process is repeated until convergence. For the photometric mapping estimation, we first tried the histogram based algorithm; ${ }^{6}$ however, we noticed that the algorithm does not perform well when there is occlusion due to saturation or motion. Therefore, we modified the algorithm to handle such cases. The original algorithm argues that two photometrically different images would have the same histogram after histogram equalization; therefore, the intensity mapping functions (IMFs) are estimated from the histogram equalization functions. To handle occlusion, we use weighted histograms, where each pixel in an image is weighted according to the likelihood of its visibility in the other image. Let $S_{i, k}$ be the set of pixels in image $I_{i}$ with value $k$ : $S_{i, k}=\left\{\mathbf{x} \mid I_{i}(\mathbf{x})=k\right\}$, and let $w_{i}(\mathbf{x})$ be the weight of $I_{i}(\mathbf{x})$, then the weighted histogram is calculated as:

$$
h_{i}(k)=\sum_{\mathbf{x} \in S_{i, k}} w_{i}(\mathbf{x}), \quad i=1,2,
$$

which is then normalized as $\bar{h}_{i}(k)=\frac{h_{i}(k)}{\sum_{k} h_{i}(k)}$.

We define $T_{i}$ as the histogram equalization function for the $i$ th image, and because histogram equalization would produce the same histograms ${ }^{6}$ we have:

$$
T_{1}\left(\bar{h}_{1}(k)\right)=T_{2}\left(\bar{h}_{2}(k)\right) .
$$

From this, the intensity mapping functions (IMFs) can be estimated as

$$
g_{i j}(\cdot)=T_{i}^{-1}\left(T_{j}(\cdot)\right), \quad(i, j)=(1,2) \text { and }(2,1) .
$$

Since it is expected that the residual between the images would be small after photometric and geometric registration, unless there is saturation or occlusion, $w_{i}(\mathbf{x})$ is chosen as an inversely proportional function of the residual. In our experiments we used a sigmoid function to determine the weights from the residual images:

$$
w_{i}(\mathbf{x})=0.5-\tan ^{-1}\left(\left(\left|r_{i}(\mathbf{x})\right|-\mu\right) / \pi \sigma\right) .
$$

This function, whose shape is controlled by the parameters $\mu$ and $\sigma$, returns a value in the range [0,1]. For small values of the residual, the output is close to 1 ; and for large values of the residual, the output approaches to 0 . (This function is arbitrarily chosen; other functions with a similar characteristics could also be used.)

Returning to our problem, we need to estimate both the motion field $\mathbf{u}(\mathbf{x})$ and the intensity mapping functions. We use the following alternating optimization algorithm:

1. Initialize: $w_{1}(\mathbf{x})=1$ and $w_{2}(\mathbf{x})=1, \forall \mathbf{x}$. $T_{2}$.

2. Calculate the weighted histograms $h_{1}$ and $h_{2}$ using (5), and the histogram equalization functions $T_{1}$ and

3. Estimate the photometric mappings $g_{12}$ and $g_{21}$ from (7).

4. Estimate the dense motion field using the photometrically mapped images. (Any optical flow algorithm can be used.)

5. Compute the residuals as in (3) and (4), and update the weights using (8).

6. Go to Step 2 unless a stopping criterion is reached. (The stopping criterion could be the number of iterations, the percentage of change in the residuals, or the percentage of change in the motion vectors, among other possibilities.) 


\section{EXPERIMENTAL RESULTS}

We have conducted a number of experiments with simulated and real data to investigate the performance of the proposed algorithm. In the first experiment (shown in Figure 1), we simulated photometric mapping on a standard test sequence, and evaluated the accuracy of our motion field estimate by comparing with the ground truth. Two images with ground truth motion field were taken from the Middlebury dataset. ${ }^{22}$ We applied gamma correction of factors $1.1,1.5$, and 2.0 to the second image to simulate different levels of photometric variations. We used a standard optical flow estimation algorithm ${ }^{23}$ to estimate the motion field. The accuracy of the motion field estimate drops significantly with increasing gamma factor. On the other hand, the proposed algorithm performs very well in all cases with good motion field and photometric mapping estimates.

The second experiment (shown in Figure 2) is the application of our method to a real imagery, where the images are captured with different exposure times and a moving camera. We created HDR images using different methods and applied tonemapping to save them as standard 8-bit RGB images. The results show that the proposed algorithm can eliminate ghosting artifacts significantly compared to no-geometric registration and a geometric registration algorithm that is available in the HDR tool we used. The third and fourth experiments (shown in figures 3 and 4) are again with real imagery, where there is local movement. Figure 5 includes a set of the motion field and the photometric mapping estimation results for the data in Figure 4. The associated visibility map indicates regions that are occluded or not accurately registered with the estimated motion.

It was observed that the estimations converge very quickly. In the experiments, we set the number of iterations to three. The computational complexity of intensity mapping function estimation is relatively small compared to the optical flow estimation; thus the time complexity for the motion vector generation depends largely upon the implementation of the dense motion field estimation algorithm used.

\section{CONCLUSIONS AND FUTURE WORK}

In this paper, we presented a method to estimate both the global photometric mapping and dense motion field in image sequences. The experiments show that even small photometric changes may significantly degrade the performance of a standard optical flow estimation method. The proposed method produces promising results with real and synthetic data. The method relies on estimation of intensity mapping functions using weighted histograms, where the weights reflect the visibility of pixels. The weights that are extracted as latent information can also be used in the HDR creation approach where weighted sum of input images is taken. There are some issues, such as the weight function selection, that need to be investigated further. Finally, we note that the algorithm could be used in other applications, including super-resolution image restoration and mosaicking, where accurate motion estimation is necessary and there are photometric variations among input images.

\section{REFERENCES}

[1] Reinhard, E., Ward, G., Pattanaik, S., and Debevec, P. in [High dynamic range imaging: acquisition, display and image-based lighting], Morgan Kaufmann (2006).

[2] Cho, W.-H. and Hong, K.-S., "Extending dynamic range of two color images under different exposures," in [Proc. Int. Conf. on Pattern Recognition], 4, 853-856 (2004).

[3] Eden, A., Uyttendaele, M., and Szeliski, R., "Seamless image stitching of scenes with large motions and exposure differences," in [Proc. Int. Conf. Computer Vision and Pattern Recognition], 2, 2498-2505 (2006).

[4] Khan, E., Akyuz, A., and Reinhard, E., "Ghost removal in high dynamic range images," in [Proc. Int. Conf. Image Processing], (2006).

[5] Jacobs, K., Loscos, C., and Ward, G., "Automatic high-dynamic range image generation for dynamic scenes," IEEE Trans. Computer Graphics and Applications 28(2), 84-93 (1996).

[6] Grossberg, M. D. and Nayar, S. K., "Determining the camera response from images: what is knowable?," IEEE Trans. Pattern Analysis and Machine Intelligence 25, 1455-1467 (November 2003).

[7] Gevrekci, M. and Gunturk, B. K., "Illumination robust interest point detection," Elsevier Computer Vision and Image Understanding (CVIU) 113, 565-571 (April 2009).

[8] Kharbat, M., Aouf, N., Tsourdos, A., and White, B., "Robust brightness description for computing optical flow," in [Proc. British Machine Vision Conference], (2008). 


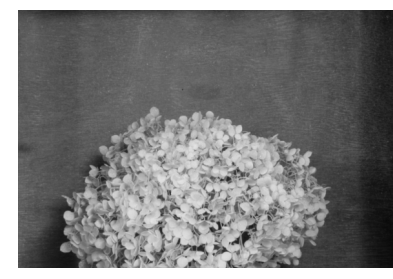

a

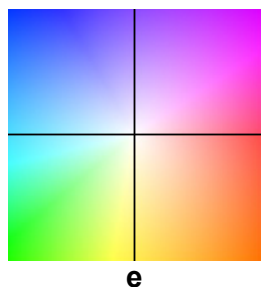

e

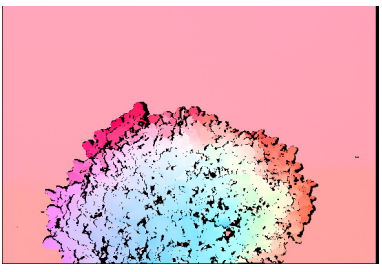

i

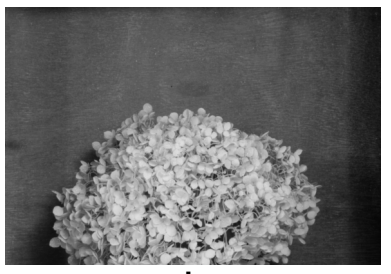

b

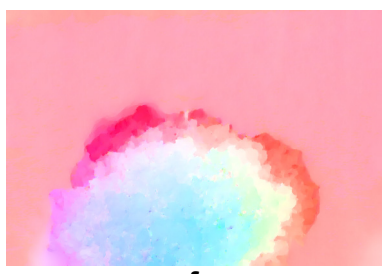

f
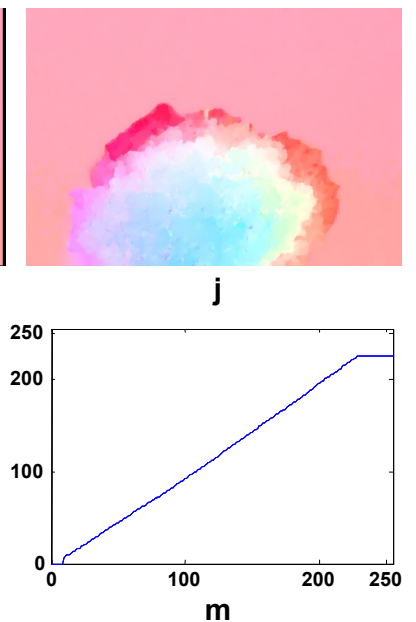

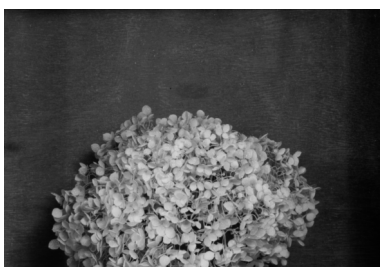

C

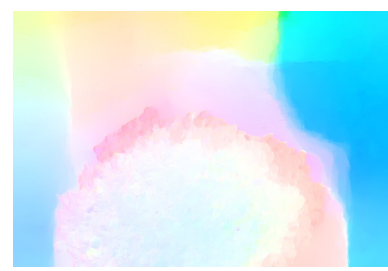

g

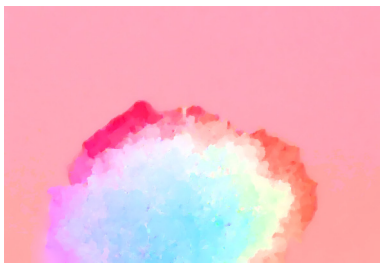

k

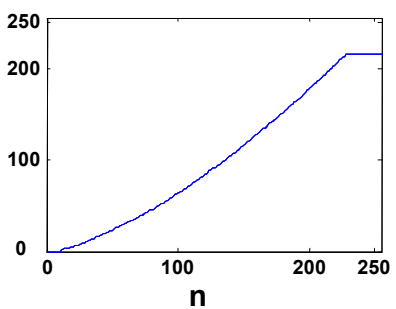

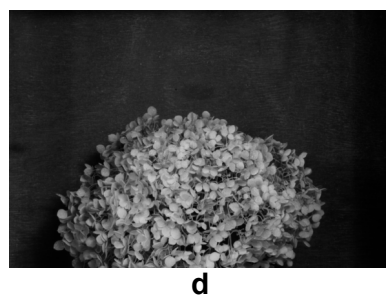

d

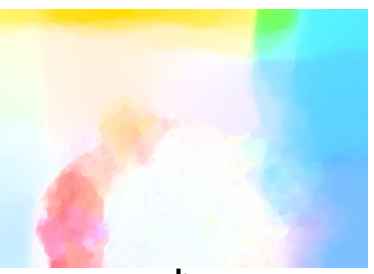

h

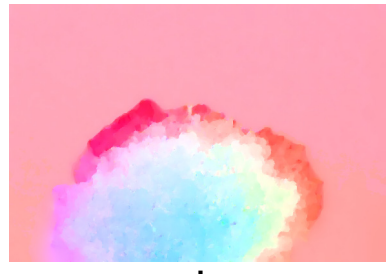

I

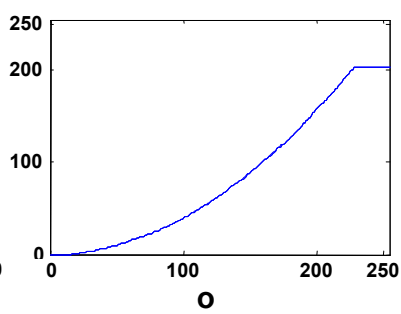

Figure 1. Motion estimation results the Middlebury Hydrangea dataset (real motion, simulated gamma). [a] Original Hydrangea Frame 10; [b] Hydrangea Frame 11 with gamma correction of 1.1; [c] Hydrangea Frame 11 with gamma correction of 1.5; [d] Hydrangea Frame 11 with gamma correction of 2.0; [e] Optical flow reference color wheel; [f-h] Motion field estimates from $\mathbf{a}$ to $\mathbf{b}, \mathbf{a}$ to $\mathbf{c}$, a to $\mathbf{d}$, respectively, with the TVL1 algorithm ${ }^{23}$ without any photometric correction; [i] Middlebury ground truth optical flow; [j-l] Motion field estimates from a to b, a to c, a to d, respectively, using the proposed method, where the TVL1 algorithm ${ }^{23}$ is for motion estimation during the iterations; [m-o] IMF estimates from $\mathbf{a}$ to $\mathbf{b}, \mathbf{a}$ to $\mathbf{c}, \mathbf{a}$ to $\mathbf{d}$, respectively, using the proposed method. 


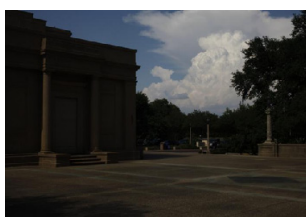

a1

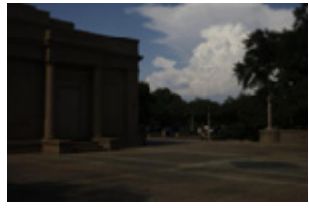

b1

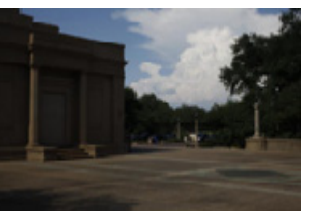

a2

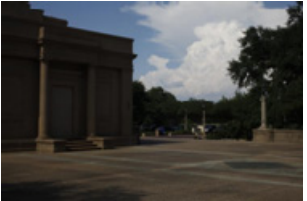

b2

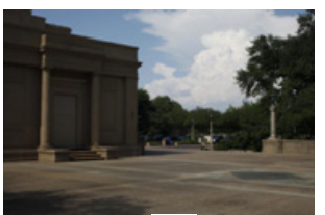

a3

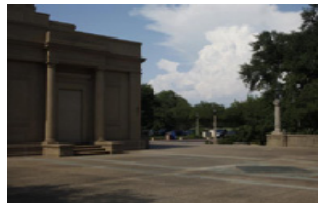

b3

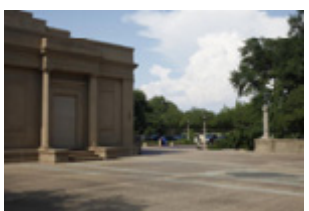

a4

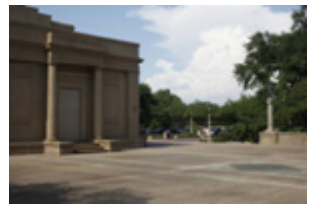

b4

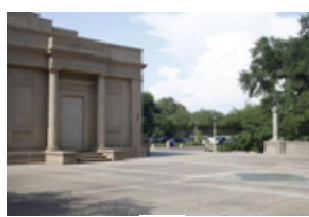

a5

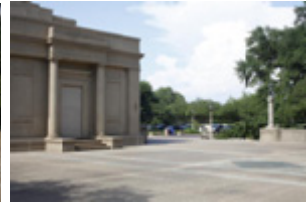

b5

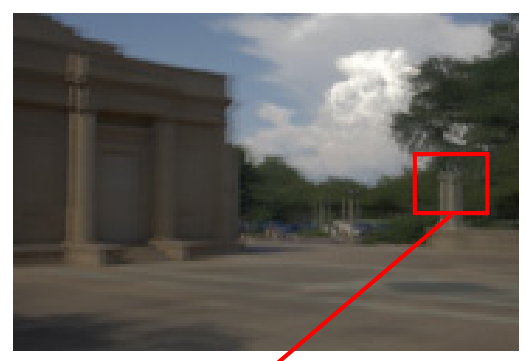

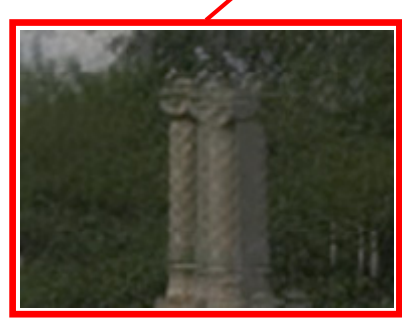

c1
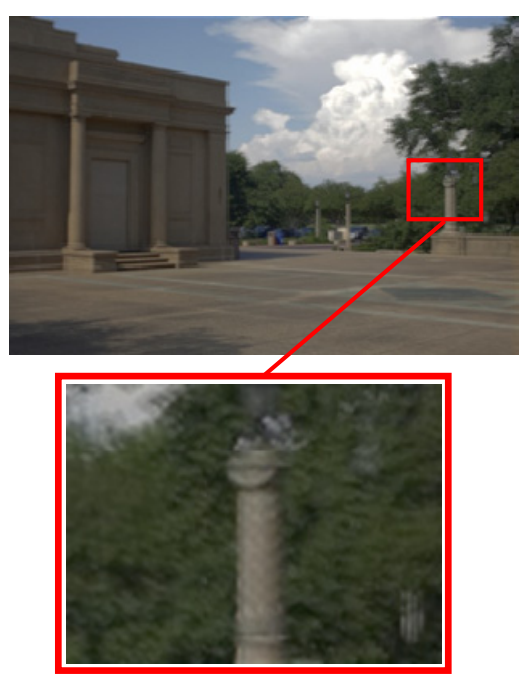

c2
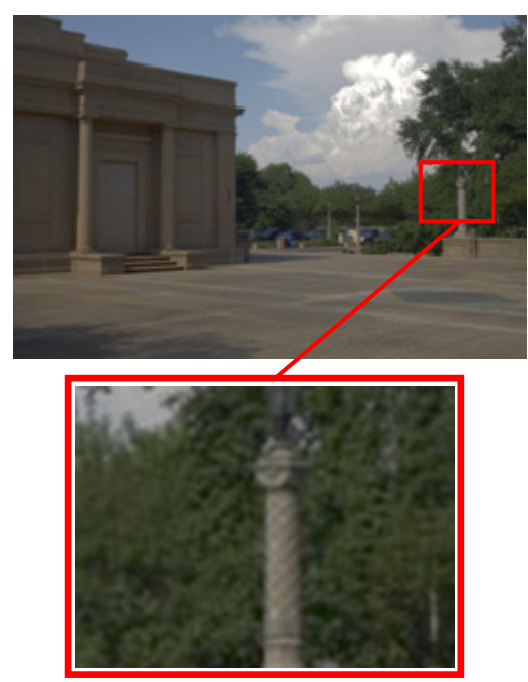

c3

Figure 2. HDR image creation. [a1 - a5] Original image sequence; [b1 - b5] Image sequence (a1-a5) geometrically registered to a1 using the proposed method; [c1] HDR result when there is no pre-alignment; [c2] HDR result after the spatial alignment that is available in QtpfsGUI (www.qtpfsgui.sourceforge.net); [c3] HDR result after the proposed method is used for motion compensation. 


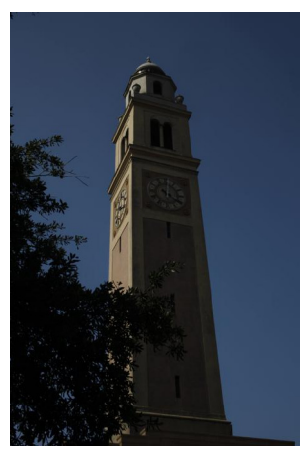

a1

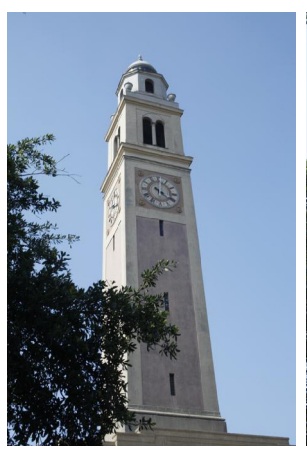

a2

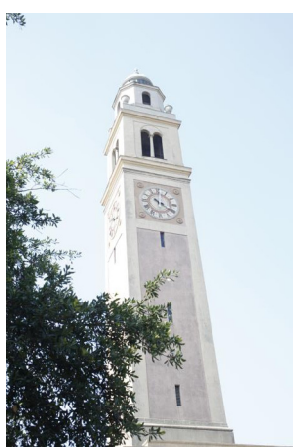

a3

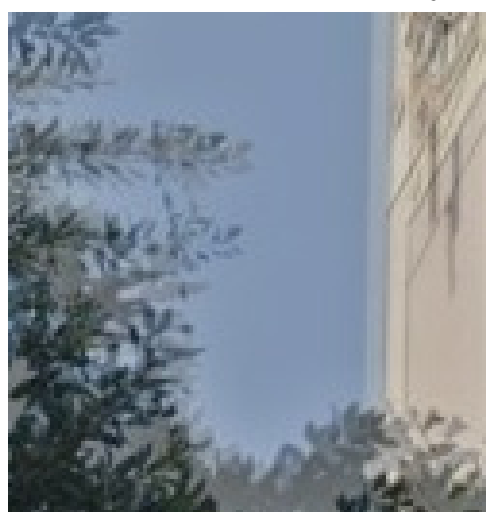

b1

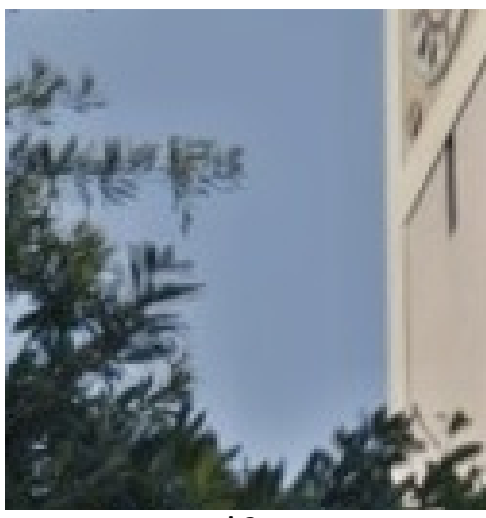

b2

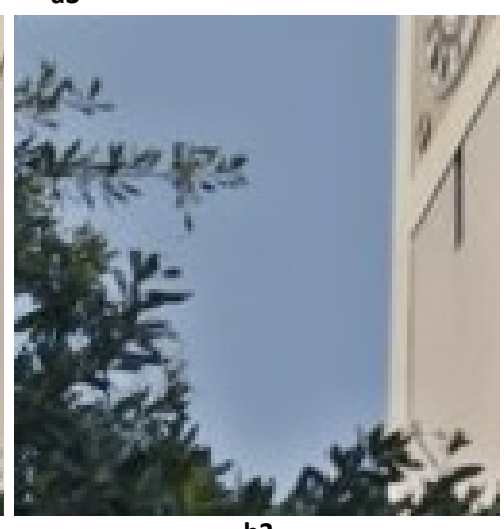

b3

Figure 3. HDR image creation. [a1 - a3] Original image sequence; [b1] HDR result (cropped) when there is no prealignment; [b2] HDR result after the spatial alignment that is available in Photomatix (www.hdrsoft.com); [b3] HDR result after the proposed method is used for motion compensation.

[9] Baker, S. and Matthews, I., "Lucas-kanade 20 years on: A unifying framework," Int. Journal of Computer Vision 56, 221-255 (February-March 2004).

[10] Bartoli, A., "Groupwise geometric and photometric direct image registration," in [IEEE Trans. Pattern Analysis and Machine Intelligence], 30(12), 2098-2108 (2008).

[11] Lucas, B. and Kanade, T., "An iterative image registration technique with an application to stereo vision," in [Proc. DARPA Image Understanding Workshop], 121-130 (1981).

[12] Kim, Y., Martinez, A. M., and Kak, A. C., "A local approach for robust optical flow estimation under varying illumination," in [Proc. British Machine Vision Conference], (2004).

[13] Kim, Y.-H., Martinez, A. M., and Kak, A. C., "Robust motion estimation under varying illumination," Image and Vision Computing 23(4), 365-375 (2005).

[14] Kim, Y. and Kak, A. C., "Error analysis of robust optical flow estimation by least median of squares methods for the varying illumination model," IEEE Trans. Pattern Analysis and Machine Intelligence 28, 1418-1435 (September 2006).

[15] Negahdaripour, S. and Yu, C., "A generalized brightness change model for computing optical flow," 2-11 (1993).

[16] Negahdaripour, S., "Revised definition of optical flow: Integration of radiometric and geometric cues for dynamic scene analysis," IEEE Trans. Pattern Analysis and Machine Intelligence 20, 961-979 (September 1998).

[17] Horn, B. and Schunck, B., "Determining optical flow," Artificial Intelligence 17, 185-203 (1981).

[18] Altunbasak, Y., Mersereau, R., and Patti, A., "A fast parametric motion estimation algorithm with illumination and lens distortion correction," IEEE Trans. Image Processing 12, 395-408 (April 2003).

[19] Periaswamy, S. and Farid, H., "Elastic registration in the presence of intensity variations," in [IEEE Trans. Pattern Analysis and Machine Intelligence], 22(7), 865-874 (2003). 


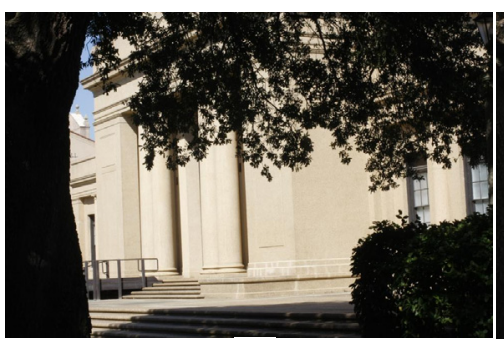

a1

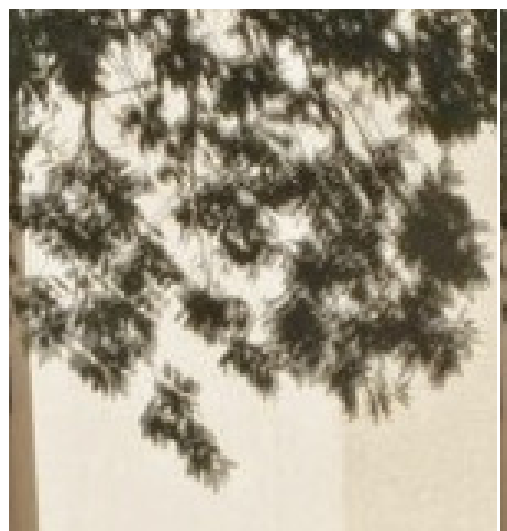

b1

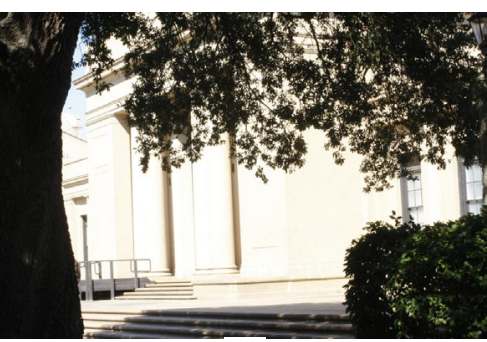

a2

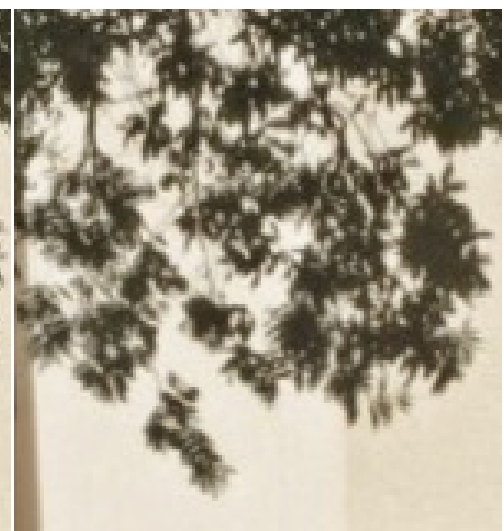

b2

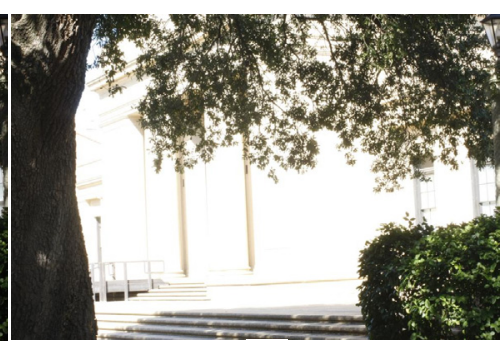

a3

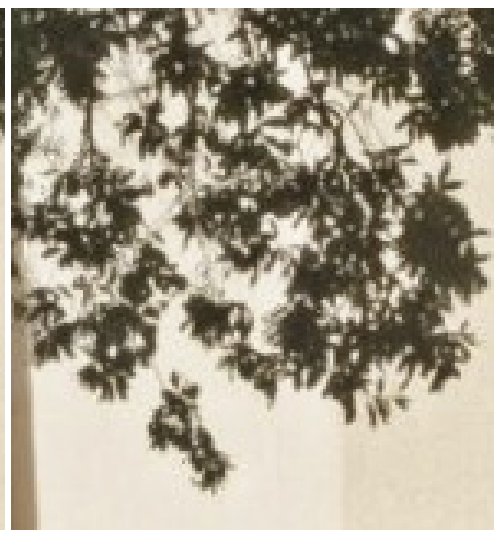

b3

Figure 4. HDR image creation. [a1 - a3] Original image sequence; [b1] HDR result (cropped) when there is no prealignment; [b2] HDR result after the alignment that is available in Photomatix (www.hdrsoft.com); [b3] HDR result after the proposed method is used for motion compensation.

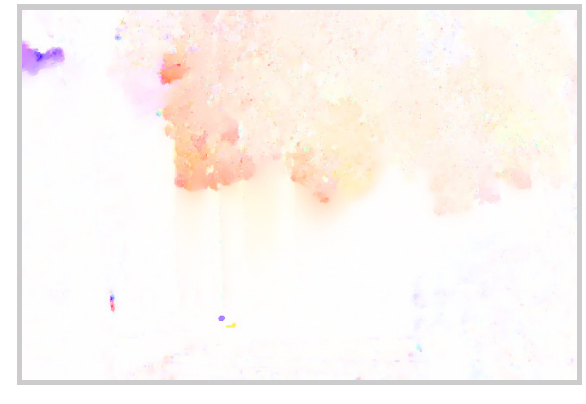

a
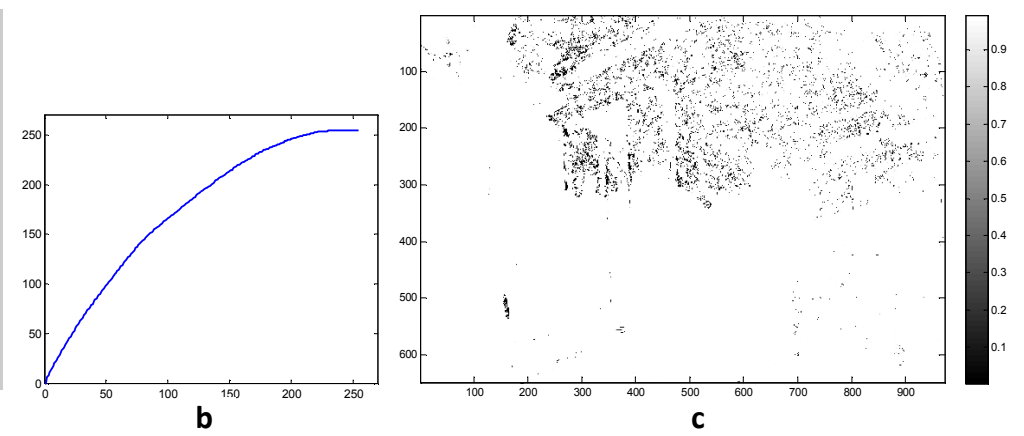

Figure 5. Estimated motion field and photometric mapping from Figure 4a1 to Figure 4a2. [a] Estimated motion field; [b] Estimated IMF; [c] Estimated weights that indicate the visibility of pixels.

[20] Aguiar, P. M. Q., "Unsupervised simultaneous registration and exposure correction," in [Proc. IEEE Int. Conf. on Image Processing], 361-364 (2006).

[21] Fouad, M., Dansereau, R., and Whitehead, A., "Geometric registration of images with arbitrarily-shaped local intensity variations from shadows," in [Proc. IEEE Int. Conf. on Image Processing], 201-204 (2009).

[22] Baker, S., Scharstein, D., Lewis, J., Roth, S., Black, M. J., and Szeliski, R., "A database and evaluation methodology for optical flow," in [Proc. IEEE Int. Conf. on Computer Vision], (2007).

[23] Zach, C., Pock, T., and Bischof, H., "A duality based approach for realtime tv-l1 optical flow," in [Annual Symposium of the German Association of Pattern Recognition], 214-223 (2007). 